\title{
Shoikhet's Conjecture and Duflo Isomorphism on (Co)Invariants ${ }^{\star}$
}

\author{
Damien CALAQUE and Carlo A. ROSSI \\ Department of Mathematics, ETH Zurich, 8092 Zurich, Switzerland \\ E-mail: damien.calaque@math.ethz.ch,carlo.rossi@math.ethz.ch
}

Received May 23, 2008, in final form August 29, 2008; Published online September 03, 2008

Original article is available at http://www.emis.de/journals/SIGMA/2008/060/

\begin{abstract}
In this paper we prove a conjecture of B. Shoikhet. This conjecture states that the tangent isomorphism on homology, between the Poisson homology associated to a Poisson structure on $\mathbb{R}^{d}$ and the Hochschild homology of its quantized star-product algebra, is an isomorphism of modules over the (isomorphic) respective cohomology algebras. As a consequence, we obtain a version of the Duflo isomorphism on coinvariants.
\end{abstract}

Key words: deformation quantization; formality theorems; cap-products; Duflo isomorphism 2000 Mathematics Subject Classification: 16E45; 16E40; 81Q30

\section{Introduction}

In his seminal paper [7] on the deformation quantization of Poisson manifolds M. Kontsevich proved that the differential graded Lie algebra (shortly, DGLA) of polydifferential operators on a smooth manifold $M$ is formal (i.e. it is quasi-isomorphic, as a DGLA, to its cohomology). As a consequence one obtains that any Poisson structure $\pi$ on $M$ can be quantized in the sense of [2], and that the Hochschild cohomology of the deformed quantized algebra is isomorphic to the Poisson cohomology of $(M, \pi)$. Moreover, it is known that the Hochschild cohomology of an associative algebra is naturally equipped with an associative cup-product; and Kontsevich proved that the mentioned isomorphism between Hochschild and Poisson cohomologies is actually multiplicative if $M=\mathbb{R}^{d}$, using a homotopy argument involving the so-called Kontsevich eye. The proof of this statement, known as the "compatibility with cup-products", has been clarified in [8], and appeared to have a surprising application to Lie theory [7] (see also [9] and [5]) in providing a new proof, together with a cohomological extension, of the famous Duflo isomorphism [6]. We recall to the reader that the result of Duflo states that the Poincaré-Birkhoff-Witt map can be modified so that it reduces to an isomorphism of algebras on invariants.

A homological version of Kontsevich's formality theorem has been formulated by B. Tsygan in [13] and proved by Shoikhet in [10] (in the case $M=\mathbb{R}^{d}$ ) and by Tsygan and Tamarkin in [12], using different approaches. It broadly states that the Hochschild chain complex of the algebra of smooth functions on $M$ is formal as a DG Lie module over the DGLA of polydifferential operators. Again, one obtains as a direct consequence of the general formalism on $L_{\infty}$-algebras, their modules and $L_{\infty}$-morphisms between them, that the Hochschild homology of the deformed quantized algebra is isomorphic to the Poisson homology of $(M, \pi)$. The present paper is mainly concerned about the multiplicativity of this isomorphism. Namely, Hochschild (resp. Poisson) homology is naturally a graded module over the Hochschild (resp. Poisson) cohomology algebra, and we prove that the isomorphism induced by the Tsygan-Shoikhet formality intertwines

\footnotetext{
${ }^{\star}$ This paper is a contribution to the Special Issue on Deformation Quantization. The full collection is available at http://www.emis.de/journals/SIGMA/Deformation_Quantization.html
} 
these module structures (both cohomology algebras being themselves isomorphic thanks to the compatibility with cup-products). We call our result the "compatibility with cap-products".

As in the cohomological situation, this compatibility with cap-products has an application to Lie theory in providing a version of the Duflo isomorphism for coinvariants.

The main goal of this paper is to present a short and comprehensible proof of this conjecture (which is a particular case of a more general result, whose detailed proof is presented in [4]). Here the proof also relies on a homotopy argument, Kontsevich's eye being replaced by the I-cube, this time being a manifold with corners of dimension 3 .

The paper is organized as follows. In Section 2 we state the main result we mentioned in this introduction. Section 3 is a brief reminder on Kontsevich's and Shoikhet's configuration spaces and their compactifications; we also mention how they are related to each other, a fact which will play a central rôle in some later computations. In Section 4 we recall the construction of Kontsevich's and Shoikhet's formality $L_{\infty}$-quasi-isomorphisms. This is the first time where the configuration spaces that were introduced in the previous section appear operatively. Section 5 is the heart of the paper. It contains the proof of the main result, for which compactified configuration spaces (and integrals over them) play a crucial rôle. We finally end the paper with the proof of a version of the Duflo isomorphism for coinvariants, which we obtain as a consequence of our main result; we only observe that we prefer to give a direct computational proof, as opposed to the proof of the same result in [10], where it was proved under the assumption that the conjecture (whose proof is the core of this paper) were true.

\section{The main result}

For the manifold $V=\mathbb{R}^{d}$, we consider the differential graded Lie algebras (shortly, DGLA) $T_{\text {poly }}^{\bullet}(V)$ and $\mathcal{D}_{\text {poly }}^{\bullet}(V)$ of polyvector vector fields on $V$ and of polydifferential operators on $V$ respectively.

Further, let $\gamma$ be a solution of the Maurer-Cartan equation in $T_{\text {poly }}^{\bullet}(V)$ of the form

$$
\gamma=\hbar \pi
$$

where $\pi$ is a bivector field and $\hbar$ a formal parameter (therefore in particular $\pi$ is a Poisson structure). The Formality Theorem of Kontsevich [7] implies that the polydifferential operator

$$
B=\mathcal{U}(\gamma)=\sum_{n \geq 1} \frac{1}{n !} \mathcal{U}_{n}(\underbrace{\gamma, \ldots, \gamma}_{n})
$$

$\mathcal{U}_{n}$ being the Taylor components of the $L_{\infty}$-quasi isomorphism between $T_{\text {poly }}^{\bullet}(V)$ and $\mathcal{D}_{\text {poly }}^{\bullet}(V)$, satisfies the Maurer-Cartan equation in the DGLA $\mathcal{D}_{\text {poly }}^{\bullet}(V) \llbracket \hbar \rrbracket$ of (series of) polydifferential operators on $V$, viewed as a subcomplex of the Hochschild cochain complex of the $\mathbb{C} \llbracket \hbar \rrbracket$-algebra $A=C^{\infty}(V) \llbracket \hbar \rrbracket$.

We denote by $\mu$ the usual multiplication on the algebra $A$ : it may be viewed as an element of $\mathcal{D}_{\text {poly }}^{\bullet}(V) \llbracket \hbar \rrbracket$ of degree 1 , and the sum $\mu+B$ specifies an associative product $\star$ on $A$, which is a deformation of the usual product on $A$, and which moreover satisfies

$$
f \star g-g \star f=2 \hbar\langle\pi, \mathrm{d} f \wedge \mathrm{d} g\rangle+\mathcal{O}\left(\hbar^{2}\right) .
$$

In other words, $\star$ is a quantization of the Poisson structure $\pi$ on $V$ in the sense of [2].

The DGLA $T_{\text {poly }}^{\bullet}(V)$ possesses an associative product $\cup$, namely the usual $\wedge$-product on $T_{\text {poly }}^{\bullet}(V)$, and a solution $\gamma$ of the Maurer-Cartan equation defines, by means of the SchoutenNijenhuis bracket ${ }^{1}$, a differential $\gamma \cdot=[\gamma$,$] on T_{\text {poly }}^{\bullet}(V)$ w.r.t. $\cup$.

\footnotetext{
${ }^{1}$ We observe that, if we follow the sign conventions of [1], we have then to modify the Schouten-Nijenhuis bracket as $[\alpha, \beta]_{\mathrm{SN}}^{\prime}=-[\beta, \alpha]_{\mathrm{SN}}$, where the Schouten-Nijenhuis bracket on the right-hand side is the usual one.
} 
The (negatively graded) deRham complex $\Omega^{-\bullet}(V)$ of differential forms on $V$ is naturally a differential graded module (shortly, DGM) over the DGLA $T_{\text {poly }}^{\bullet}(V)$ : the extensions of the Lie derivative $\mathrm{L}$ by means of the Cartan formula and of the contraction operator $\iota$ define respectively a differential $\mathrm{L}_{\gamma}$, for $\gamma$ as in (1), and an action $\cap$ of $T_{\text {poly }}^{\bullet}(V)$ on $\Omega^{-\bullet}(V)$.

On the other hand, for $\gamma$ as above, there is a product $\cup$ (of degree 1 ) on the (shifted by 1 ) Hochschild cochain complex $C^{\bullet}(A, A)[1]$ of $A$ with values in $A$,

$$
(\varphi \cup \psi)\left(a_{1}, \ldots, a_{p+q}\right)=\varphi\left(a_{1}, \ldots, a_{p}\right) \star \psi\left(a_{p+1}, \ldots, a_{p+q}\right) .
$$

Additionally, the Hochschild differential $\mathrm{d}_{\mathrm{H}}$ on the Hochschild cochain complex of $A$ is modified to the Hochschild differential $\mathrm{d}_{\mathrm{H}, \star}$ w.r.t. $\star$. All these structures descend to the subcomplex of polydifferential operators on $V$.

For the algebra $A$, we consider the (negatively graded) Hochschild chain complex $C_{-\bullet}(A, A)$. For $\gamma$ as (1), there is an action of $C^{\bullet}(A, A)[1]$ on $C_{-\bullet}(A, A)$ via

$$
\varphi \cap\left(a_{0}\left|a_{1}\right| \cdots \mid a_{n}\right)=\left(a_{0} \star \varphi\left(a_{1}, \ldots, a_{m}\right)\left|a_{m+1}\right| \cdots \mid a_{n}\right),
$$

if $m \leq n$; if $m>n$, the action is trivial. Furthermore, we also have the differential $\mathrm{b}_{\star}$ on $C_{-\bullet}(A, A)$, which modifies the usual Hochschild differential b on $C_{-\bullet}(A, A)$. The previous formula defines also an action of the DGLA of polydifferential operators on $V$ on $C_{-\bullet}(A, A)$.

We denote by $\mathcal{S}_{n}, n \geq 0$, the Taylor components of the $L_{\infty}$-quasi-isomorphism $\mathcal{S}$ from the $L_{\infty}$-module $C_{-\bullet}(A, A)$ to the DGM $\Omega^{\bullet}(V) \llbracket \hbar \rrbracket$, both over $T_{\text {poly }}^{\bullet}(V)$, constructed in [10]. The DGM structure of $\Omega^{\bullet}(V) \llbracket \hbar \rrbracket$ over $T_{\text {poly }}^{\bullet}(V)$ comes from the Lie derivative of polyvector fields on differential forms, while, as shown in [10], composition of the $L_{\infty}$-quasi-isomorphism $\mathcal{U}$ with the action $\mathrm{L}$ of Hochschild cochains on $A$ on Hochschild chains gives $C_{-\bullet}(A, A)$ the structure of an $L_{\infty}$-module over $T_{\text {poly }}^{\bullet}(V)$.

For a solution $\gamma$ of the Maurer-Cartan equation as in (1), we consider the following linear maps:

$$
\begin{aligned}
\mathcal{U}_{\gamma}(\alpha) & =\sum_{n \geq 0} \frac{1}{n !} \mathcal{U}_{n+1}(\alpha, \underbrace{\gamma, \ldots, \gamma}_{n}), \quad \text { resp. } \\
\mathcal{S}_{\gamma}(c) & =\sum_{n \geq 0} \frac{1}{n !} \mathcal{S}_{n}(\underbrace{\gamma, \ldots, \gamma}_{n}, c),
\end{aligned}
$$

for a general polyvector field $\alpha$ on $V$, resp. Hochschild chain $c$ on $A$.

Theorem 1. For a solution $\gamma$ of the Maurer-Cartan equation as in (1), (2) is a quasi-isomorphism of complexes

$$
\mathcal{U}_{\gamma}:\left(T_{\text {poly }}^{\bullet}(V) \llbracket \hbar \rrbracket, \gamma \cdot\right) \rightarrow\left(\mathcal{D}_{\text {poly }}^{\bullet}(V) \llbracket \hbar \rrbracket, \mathrm{d}_{\mathrm{H}, \star}\right),
$$

which additionally preserves the products in the corresponding cohomologies,

$$
\left[\mathcal{U}_{\gamma}([\alpha] \cup[\beta])\right]=\left[\mathcal{U}_{\gamma}([\alpha]) \cup \mathcal{U}_{\gamma}([\beta])\right],
$$

square brackets denoting cohomology classes.

We refer to $[7,8]$ for the proof of Theorem 1 . The main result of this paper is the proof of Conjecture 3.5.3.1 in [10], which we may state in the following

Theorem 2. For a solution $\gamma$ of the Maurer-Cartan equation as in (1), (3) is a quasi-isomorphism of complexes

$$
\mathcal{S}_{\gamma}:\left(C_{-\bullet}(A, A), \mathrm{b}_{\star}\right) \rightarrow\left(\Omega^{\bullet}(V) \llbracket \hbar \rrbracket, \mathrm{L}_{\gamma}\right)
$$

and additionally preserves the action of $T_{\text {poly }}^{\bullet}(V)$ in the corresponding cohomologies,

$$
\left[[\alpha] \cap \mathcal{S}_{\gamma}([c])\right]=\left[\mathcal{S}_{\gamma}\left(\mathcal{U}_{\gamma}([\alpha]) \cap[c]\right)\right],
$$

with the previous notation for cohomology classes. 


\section{Configuration spaces and their compactifications}

We briefly discuss in this Section configuration spaces of $i$ ) points in the complex upper-half plane $\mathcal{H}$ and on the real axis $\mathbb{R}$, and $i i$ ) points in the interior of the punctured unit disk $D$ and on the boundary $S^{1}$, and their compactifications à la Fulton-MacPherson.

\subsection{Configuration spaces of points in the upper half-plane}

For a pair of non-negative integers $(n, m)$, the (open) configuration space $C_{n, m}^{+}$is defined as

$$
C_{n, m}^{+}=\left\{\left(p_{1}, \ldots, p_{n}, q_{1}, \ldots, q_{m}\right) \in \mathcal{H}^{n} \times \mathbb{R}^{m}: p_{i} \neq p_{j}, i \neq j, q_{1}<\cdots<q_{m}\right\} / G_{2},
$$

where $G_{2}$ is the semidirect product $\mathbb{R}^{+} \ltimes \mathbb{R}$, acting via rescalings and translations. If $2 n+m-2 \geq$ $0, C_{n, m}^{+}$is a smooth real manifold of dimension $2 n+m-2$. We may consider more general configuration spaces $C_{A, B}^{+}$, where $A$ is any finite set and $B$ is any ordered finite set.

The configuration space $C_{n}$ is defined as

$$
C_{n}=\left\{\left(p_{1}, \ldots, p_{n}\right) \in \mathbb{C}^{n}: p_{i} \neq p_{j}, i \neq j\right\} / G_{3}
$$

where $G_{3}$ is the semidirect product $\mathbb{R}^{+} \ltimes \mathbb{C}$, acting via rescalings and complex translations. If $2 n-3 \geq 0, C_{n}$ is a smooth real manifold of dimension $2 n-3$. Again, we may consider more general configuration spaces $C_{A}$, for any finite set $A$.

Both configuration spaces $C_{A, B}^{+}$and $C_{A}$ are orientable, see e.g. [1].

Configuration spaces $C_{A, B}^{+}$and $C_{A}$ admit compactifications à la Fulton-MacPherson, denoted by $\mathcal{C}_{A, B}^{+}$and $\mathcal{C}_{A}$ respectively: they are smooth manifolds with corners and we refer to $[7,8,3]$ and [4] for their explicit constructions.

\subsection{Configuration spaces of points in the punctured disk}

As in Subsection 3.1, for a pair of non-negative integers $(n, m), m \geq 1$, the (open) configuration space $D_{n, m}^{+}$is defined as

$$
D_{n, m}^{+}=\left\{\left(p_{1}, \ldots, p_{n}, q_{1}, \ldots, q_{m}\right) \in\left(D^{\times}\right)^{n} \times\left(S^{1}\right)^{m}:\left\{\begin{array}{l}
p_{i} \neq p_{j}, \quad i \neq j, \\
q_{1}<\cdots<q_{m}<q_{1},
\end{array}\right\} / S^{1},\right.
$$

where $D^{\times}$denotes the punctured unit disk, and where we introduced a cyclic order on $S^{1}$; the group $S^{1}$ acts by rotations. If $2 n+m-1 \geq 0, D_{n, m}^{+}$is a smooth real manifold of dimension $2 n+m-1$. As before, we may consider configuration spaces $D_{A, B}^{+}$, where $A$ is any finite set and $B$ is any cyclically ordered finite set. When $|B|=1$, we omit the superscript + .

For a positive integer $n$, we consider the configuration space

$$
D_{n}=\left\{\left(p_{1}, \ldots, p_{n}\right) \in\left(\mathbb{C}^{\times}\right)^{n}: p_{i} \neq p_{j}, i \neq j\right\} / \mathbb{R}^{+},
$$

where $\mathbb{R}^{+}$acts by rescaling. It is obviously a smooth real manifold of dimension $2 n-1$, when $2 n-1 \geq 0$. We may consider configuration spaces $D_{A}$, with $A$ any finite set.

Finally, $D_{A, B}^{+}$and $D_{A}$ are orientable, by the same arguments as in [1].

Configuration spaces $D_{A, B}^{+}$and $D_{A}$, admit compactifications à la Fulton-MacPherson, denoted by $\mathcal{D}_{A, B}^{+}$and $\mathcal{D}_{A}$ respectively, which are smooth manifolds with corners.

Being $\mathcal{D}_{A, B}^{+}$a stratified space, its boundary strata of codimension 1 are given in the following list: 
i) There is a subset $A_{1}$ of $A$, obeying $1 \leq\left|A_{1}\right| \leq|A|$, such that

$$
\partial_{A_{1}, 0} \mathcal{D}_{A, B}^{+} \cong \mathcal{D}_{A_{1}} \times \mathcal{D}_{A \backslash A_{1}, B}^{+}
$$

Clearly, $2\left|A_{1}\right|-1 \geq 0$ and $2\left(|A|-\left|A_{1}\right|\right)+|B|-1 \geq 0$. Intuitively, this corresponds to the situation, where points in $D^{\times}$labelled by $A_{1}$ collapse together to the origin.

ii) There is a subset $A_{1}$ of $A$, obeying $2 \leq\left|A_{1}\right| \leq|A|$, such that

$$
\partial_{A_{1}} \mathcal{D}_{A, B}^{+} \cong \mathcal{C}_{A_{1}} \times \mathcal{D}_{A \backslash A_{1} \sqcup\{\bullet\}, B}^{+} .
$$

We must impose $2\left|A_{1}\right|-3 \geq 0$ and $2\left(|A|-\left|A_{1}\right|+1\right)+|B|-1 \geq 0$. Intuitively, this corresponds to the situation, where points in $D^{\times}$labelled by $A_{1}$ collapse together to a single point in $D^{\times}$labelled by $\bullet$.

iii) Finally, there is a subset $A_{1}$ of $A$ and an ordered subset $B_{1}$ of successive elements of $B$, obeying $0 \leq\left|A_{1}\right| \leq|A|$ and $2 \leq\left|B_{1}\right| \leq|B|$, such that

$$
\partial_{A_{1}, B_{1}} \mathcal{D}_{A, B}^{+} \cong \mathcal{C}_{A_{1}, B_{1}}^{+} \times \mathcal{D}_{A \backslash A_{1}, B \backslash B_{1} \sqcup\{\bullet\}}^{+} \cdot
$$

We impose $2\left|A_{1}\right|+\left|B_{1}\right|-2 \geq 0$ and $2\left(|A|-\left|A_{1}\right|\right)+\left(|B|-\left|B_{1}\right|+1\right)-1 \geq 0$. Intuitively, this corresponds to the situation, where points in $D^{\times}$labelled by $A_{1}$ and points in $S^{1}$ labelled by $B_{1}$ collapse together to a single point in $S^{1}$ labelled by $\bullet$.

\subsection{An identification between compactified configuration spaces}

We may use the action of $S^{1}$ to construct a section of $D_{A, B}^{+}$, namely we fix one point $\circ$ in $S^{1}$ to 1. This section is diffeomorphic, by means of the Möbius transformation

$$
\psi: \mathcal{H} \sqcup \mathbb{R} \longrightarrow D \sqcup S^{1} \backslash\{1\} ; \quad z \longmapsto \frac{z-\mathrm{i}}{z+\mathrm{i}},
$$

where $D$ is the unit disk, to a smooth section of $C_{A \sqcup\{\bullet\}, B \backslash\{\circ\}}^{+}$, given by fixing one point $\bullet$ in the complex upper half-plane $\mathcal{H}$ to i by means of the action of $G_{2}$.

Then, the compactified configuration space $\mathcal{D}_{A, B}^{+}$can be identified with $\mathcal{C}_{A \sqcup\{\bullet\}, B \backslash\{\circ\}}^{+}$, and we observe that the cyclic order on the points in $S^{1}$ translates naturally into an order on the points on the real axis $\mathbb{R}$.

We further consider the manifold $D_{A}$, and notice the identification $D_{A} \cong C_{A \sqcup\{\bullet\}}$ : to be more precise, by means of complex translation, we may put one point $\bullet$ in $C_{n+1}$ at the origin, and using rescalings, one can put the remaining points in the punctured unit disk with boundary. Analogously as before, the compactification $\mathcal{D}_{A}$ of $D_{A}$ can be identified with $\mathcal{C}_{A \sqcup\{\bullet\}}$.

We consequently identify the codimension 1 boundary strata of $\mathcal{D}_{A, B}^{+}$with those of $\mathcal{C}_{A \sqcup\{\bullet\}, B \backslash\{0\}}^{+}$ (higher codimension can be worked out along the same lines very easily):

i) A boundary stratum as in (4) corresponds to the situation, where points labelled by $A_{1} \sqcup\{\bullet\}$ collapse together to a single point in $\mathcal{H}$, which takes the rôle of the marked point $\bullet$.

ii) A boundary stratum as in (5) corresponds to the situation, where points labelled by $A_{1}$ collapse together to a single point in $\mathcal{H}$, which will not be the new marked point $\bullet$.

iii $i_{1}$ ) A boundary stratum as in (6), where $\circ \notin B_{1}$, corresponds to the situation, where points labelled by $A_{1} \sqcup B_{1}$ collapse to a single point in $\mathbb{R}$, which will not be the new marked point $\circ$.

$\left.i i i_{2}\right)$ Finally, a boundary stratum as in (6), where $\circ \in B_{1}$, corresponds to the situation, where points labelled by the set $A \backslash A_{1} \sqcup\{\bullet\} \sqcup B \backslash B_{1}$ collapse to a single point in $\mathbb{R}$, which will be the new marked point $\circ$. 


\section{Explicit formulæ for the formality morphism for cochains and chains}

Here is a short review of the formulæ we will need to construct the aforementioned $L_{\infty}$-quasiisomorphisms $\mathcal{U}$ and $\mathcal{S}$.

\subsection{The $L_{\infty}$-quasi-isomorphism $\mathcal{U}$}

For the sake of simplicity, we denote by $[n]$, for a positive integer $n$, the set $\{1, \ldots, n\}$. For any pair of non-negative integers $(n, m)$, such that $2 n+m-2 \geq 0$, an admissible graph $\Gamma$ of type $(n, m)$ is by definition a directed graph with labels obeying the following requirements:

i) The set of vertices $V_{\Gamma}$ is given by $[n] \sqcup[m]$; vertices labelled by integers in $[n]$, resp. $[m]$, are called vertices of the first, resp. second type; further, the labelling of vertices of the first type specifies an order on them. The set of vertices factorizes into $V_{\Gamma}=V_{\Gamma}^{1} \sqcup V_{\Gamma}^{2}$, where $V_{\Gamma}^{1}$, resp. $V_{\Gamma}^{2}$, is the set of vertices of the first type, resp. second type.

ii) Every edge in $E_{\Gamma}$ starts at some vertex of the first type; there is at most one edge between any two distinct vertices of $\Gamma$; no edge starts and ends at the same vertex.

For a given vertex $v$ of $\Gamma$, we denote by $\operatorname{star}(v)$ the subset of $E_{\Gamma}$ of edges starting at $v$ : then, we assume that, for any vertex of the first type $v$ of $\Gamma$, the elements of $\operatorname{star}(v)$ are labelled as

$\left(e_{v}^{1}, \ldots, e_{v}^{|\operatorname{star}(v)|}\right)$. By definition, the valence of a vertex $v$ is the cardinality of the star of $v$. The set of admissible graphs of type $(n, m)$ is denoted by $\mathcal{G}_{n, m}$.

We also need the following lemma, borrowed from [7], to which we also refer for a more careful explanation of the origins of the form $\omega$.

Lemma 1. There exists a smooth 1-form $\omega$ on $\mathcal{C}_{2,0}$, with the following properties:

i) The restriction of $\omega$ to the boundary stratum $\mathcal{C}_{2}=S^{1}$ equals the deRham differential of the angle function measured in counterclockwise direction from the positive imaginary axis.

ii) The restriction of $\omega$ to $\mathcal{C}_{1,1}$, where the first point in the complex upper half-plane goes to the real axis, vanishes.

For any pair of non-negative integers $(n, m)$, such that $2 n+m-2 \geq 0$, there are natural smooth projections from $\mathcal{C}_{n, m}^{+}$onto $\mathcal{C}_{2,0}$ (provided $n \geq 2$ ) or onto $\mathcal{C}_{1,1}$ (if $n, m \geq 1$ ), extending the natural projections on the open configuration spaces.

To an admissible graph $\Gamma$ of type $(n, m)$ is associated its Kontsevich's weight $W_{\Gamma}$ via

$$
W_{\Gamma}=\int_{\mathcal{C}_{n, m}^{+}} \bigwedge_{e \in E_{\Gamma}} \omega_{e}=\int_{\mathcal{C}_{n, m}^{+}} \omega_{\Gamma},
$$

where, for an edge $e$ of $\Gamma, \omega_{e}$ denotes the pull-back of $\omega$ to $\mathcal{C}_{n, m}^{+}$via the projection $\pi_{e}$ from $\mathcal{C}_{n, m}^{+}$ onto $\mathcal{C}_{2,0}$, onto the pair of points labelled by the endpoints of $e$. The labelling of $\Gamma$ specifies an order of the forms $\omega_{e}$ in the above product.

To an admissible graph $\Gamma$ of type $(n, m)$, to $n$ polyvector fields $\gamma_{1}, \ldots, \gamma_{n}$ and to $m$ functions $f_{1}, \ldots, f_{m}$ on $V$, such that $|\operatorname{star}(k)|=\left|\gamma_{k}\right|+1, k=1, \ldots, n$, we associate a function

$$
U_{\Gamma}\left(\gamma_{1}, \ldots, \gamma_{n}\right)\left(f_{1}, \ldots, f_{m}\right)
$$

by the following rule: to a vertex $v$ of the first type, resp. second type, we associate the polyvector field $\gamma_{v}$, resp. the function $f_{v}$. For a function $I$ from $E_{\Gamma}$ to $[d]$, we associate to the vertex $v$ of the first type, resp. second type, the function

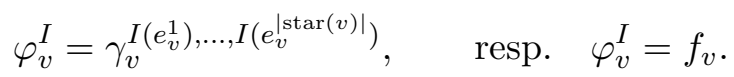


with the same notations as before. The function $I$ labels edges of $\Gamma$ by (standard) coordinates of $V$. Then, we have the following assignment, for an admissible graph $\Gamma$ of type $(n, m)$ :

$$
U_{\Gamma}\left(\gamma_{1}, \ldots, \gamma_{n}\right)\left(f_{1}, \ldots, f_{m}\right)=\sum_{I: E_{\Gamma} \rightarrow[d]} \prod_{v \in V_{\Gamma}}\left(\prod_{e \in E_{\Gamma}: e=(*, v)} \partial_{I(e)}\right) \varphi_{v}^{I} .
$$

It is clear that $U_{\Gamma}$, with $\Gamma$ as above, maps $n$ polyvector fields $\left\{\gamma_{1}, \ldots, \gamma_{n}\right\}$ to a polydifferential operator of (shifted) degree $m-1$ by its very construction.

Finally, we define the $n$-th Taylor component $\mathcal{U}_{n}$ of Kontsevich's $L_{\infty}$-quasi-isomorphism $\mathcal{U}$ by combining (7) and (8), namely

$$
\mathcal{U}_{n}=\sum_{m \geq 0} \sum_{\Gamma \in \mathcal{G}_{n, m}} W_{\Gamma} U_{\Gamma}
$$

Theorem 3 (Kontsevich). The Taylor components (9) combine to an $L_{\infty}$-quasi-isomorphism

$$
\mathcal{U}: T_{\text {poly }}^{\bullet}(V) \rightarrow \mathcal{D}_{\text {poly }}^{\bullet}(V),
$$

of $L_{\infty}$-algebras, whose first order Taylor component reduces to the Hochschild-Kostant-Rosenberg quasi-isomorphism in cohomology.

For a proof of Theorem 3, we refer to [7].

\subsection{The $L_{\infty}$-quasi-isomorphism $\mathcal{S}$}

An admissible graph of type $(n, m)$, for any two non-negative integers such that $2 n+m-1 \geq 0$, is a directed labelled graph $\Gamma$ as in Subsection 4.1, with the only difference that there is a special vertex, labelled by 0 , from which edges can only depart; the vertex 0 belongs neither to vertices of the first type nor of the second type. The other requirements and notations from Subsection 4.1 remain unaltered. The set of admissible graphs of this kind of type $(n, m)$ is denoted by $\mathcal{G}_{n, m, 0}$.

A (partial) counterpart of Lemma 1 in this framework is the following lemma.

We define a smooth 1 -form on the configuration space $C_{3,0}$ via

$$
\varphi_{D}(p, q, r)=\varphi(q, r)-\varphi(q, p)
$$

for any three pairwise distinct points $p, q, r$ in $\mathcal{H} \sqcup \mathbb{R}$ : we then set $\omega_{D}=\mathrm{d} \varphi_{D}$.

Lemma 2. The 1-form $\omega_{D}$ extends to a smooth 1-form on $\mathcal{C}_{3,0}$, with the following properties:

i) its restriction to $\mathcal{C}_{2,1}$, when $q$ approaches the real axis, vanishes;

ii) its restriction to $\mathcal{C}_{2,0} \times \mathcal{C}_{1,1}$, when $p$ and $q$ collapse together to the real axis, equals $-\pi_{1}^{*} \omega$;

iii) its restriction to $\mathcal{C}_{2} \times \mathcal{C}_{2,0}$, when $p$ and $q$ collapse together in the upper half-plane, equals $\pi_{2}^{*} \omega-\pi_{1}^{*} \omega$

$i v)$ its restriction to $\mathcal{C}_{2,0} \times \mathcal{C}_{1,1}$ (resp. $\left.\mathcal{C}_{2} \times \mathcal{C}_{2,0}\right)$, when $p$ and $r$ collapse together to the real axis (resp. in the upper half-plane), vanishes;

$v$ ) its restriction to $\mathcal{C}_{2,0} \times \mathcal{C}_{1,1}$, when $q$ and $r$ collapse together to the real axis, equals $\pi_{1}^{*} \omega$;

vi) its restriction to $\mathcal{C}_{2} \times \mathcal{C}_{2,0}$, when $q$ and $r$ collapse together in the upper half-plane, equals $\pi_{1}^{*} \omega-\pi_{2}^{*} \omega$.

As above, we define a Shoikhet's weight associated to a graph without loop $\Gamma$ with $m+n+1$ vertices labelled by $\mathcal{V}(\Gamma):=\{0, \ldots, n, \overline{1}, \ldots, \bar{m}\}$. To any edge $e=(i, j) \in \mathcal{E}(\Gamma)$, we associate a smooth 1 -form $\omega_{D, e}$ on $\mathcal{D}_{n, m}^{+}$by the following rules: 
- if neither $i$ nor $j$ lies in $\{0, \overline{1}\}$, then $\omega_{D, e}:=\pi_{(0, i, j)}^{*} \omega_{D}$, where

$$
\pi_{(0, i, j)}: \mathcal{D}_{n, m}^{+} \cong \mathcal{C}_{n+1, m-1}^{+} \longrightarrow \mathcal{C}_{3,0}, \quad\left[\left(z_{0}, \ldots, z_{n}, z_{\overline{2}}, \ldots, z_{\bar{m}}\right)\right] \longmapsto\left[\left(z_{0}, z_{i}, z_{j}\right)\right]
$$

- if $i=0$ and $j \neq 1$, then $\omega_{D, e}:=\pi_{(i, j)}^{*} \omega$, where

$$
\pi_{(i, j)}: \mathcal{D}_{n, m}^{+} \cong \mathcal{C}_{n+1, m-1}^{+} \longrightarrow \mathcal{C}_{2,0}, \quad\left[\left(z_{0}, \ldots, z_{n}, z_{\overline{2}}, \ldots, z_{\bar{m}}\right)\right] \longmapsto\left[\left(z_{i}, z_{j}\right)\right] ;
$$

- if $j=1$ and $i \neq 0$, then $\omega_{D, e}:=\mathrm{p}_{(i, j)}^{*} \omega$, where

$$
\mathrm{p}_{(i, j)}: \mathcal{D}_{n, m}^{+} \longrightarrow \mathcal{D}_{1,1} \cong \mathcal{C}_{2,0}, \quad\left[\left(z_{1}, \ldots, z_{n}, z_{\overline{1}}, \ldots, z_{\bar{m}}\right)\right] \longmapsto\left[\left(z_{i}, z_{j}\right)\right] ;
$$

- if $i=1$ or $j=0$ or $(i, j)=(0,1)$, then $\omega_{D, e}=0$.

Then, as above,

$$
\omega_{D, \Gamma}:=\bigwedge_{e \in \mathcal{E}(\Gamma)} \omega_{D, e}
$$

defines a differential form on $\mathcal{D}_{n, m}^{+}$.

Definition 1. The Shoikhet weight $W_{D, \Gamma}$ of the directed graph $\Gamma$ is

$$
W_{D, \Gamma}:=\int_{\mathcal{D}_{n, m}^{+}} \omega_{D, \Gamma}
$$

We consider an admissible graph in $\mathcal{G}_{n, m, 0}$, such that $|\operatorname{star}(0)|=l$. To $n$ polyvector fields $\left\{\gamma_{1}, \ldots, \gamma_{n}\right\}$ on $V$, such that $|\operatorname{star}(k)|=\left|\gamma_{k}\right|+1, k=1, \ldots, n$, and to a Hochschild chain $c=\left(a_{0}\left|a_{1}\right| \cdots \mid a_{m-1}\right)$ of degree $-m+1$, we associate an $l$-form on $V$ (whose actual degree is $-l$, following the grading introduced in [10]) via

$$
\left\langle\alpha, S_{\Gamma}\left(\gamma_{1}, \ldots, \gamma_{n}, c\right)\right\rangle=U_{\Gamma}\left(\alpha, \gamma_{1}, \ldots, \gamma_{n}\right)\left(a_{0}, \ldots, a_{n}\right) .
$$

Finally, the $n$-th Taylor component $\mathcal{S}_{n}$ of the $L_{\infty}$-quasi-isomorphism $\mathcal{S}$ from the $L_{\infty}$-module $C_{-\bullet}(A, A)$ to the $L_{\infty}$-module $\Omega^{-\bullet}(V)$ over $T_{\text {poly }}^{\bullet}(V)$ is given by

$$
\mathcal{S}_{n}=\sum_{m \geq 1} \sum_{\Gamma \in \mathcal{G}_{n, m, 0}} W_{\Gamma, D} S_{\Gamma}
$$

Theorem 4 (Shoikhet). The Taylor components (10) combine to an $L_{\infty}$-quasi-isomorphism

$$
\mathcal{S}: C_{-\bullet}(A, A) \rightarrow \Omega^{-\bullet}(V),
$$

of $L_{\infty}$-modules over $T_{\text {poly }}^{\bullet}(V)$, whose 0 -th order Taylor component reduces to the HochschildKostant-Rosenberg quasi-isomorphism in homology.

We refer to [10] for the proof of Theorem 4.

\section{Compatibility between the actions of polyvector fields on forms and Hochschild chains}

In this Section we sketch the proof of Theorem 2, Section 2, whose strategy owes to the homotopy argument used in [7,8]; for a more detailed version of the proof of Theorem 2 in an even more general case, we refer to [4].

We must consider separately the case, where $\mathcal{S}_{\gamma}$ acts on Hochschild chains $i$ ) of degree $m=0$ and $i i$ ) of degree $-m \leq-1$ : as we will soon notice, the geometric aspects of the two cases are quite different. 


\subsection{The space $\mathcal{D}_{1,1}$ and Hochschild chains of degree 0}

By the definition of the action $\cap$, if $c$ has degree 0 , the only Hochschild cochains acting on $c$ non-trivially must be functions, in which case the action is simply multiplication on the right w.r.t. the product $\star$.

We consider the curve $\ell$ on the configuration space $\mathcal{D}_{1,1}$, with initial point on $\alpha$, and final point $b$, which corresponds to the following embedding of the open unit interval into $D_{1,1}$ :
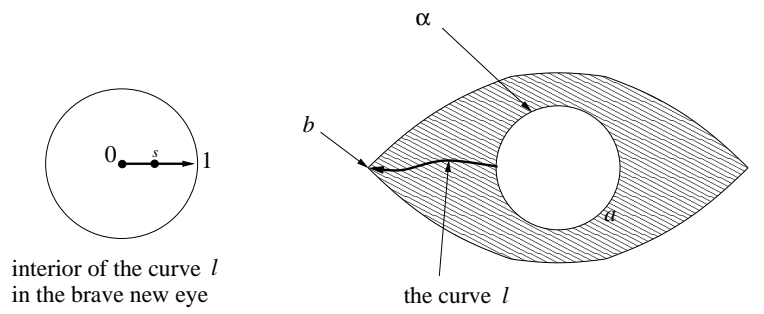

Figure 1. The curve $\ell$ in $\mathcal{D}_{1,1}$.

Since $\mathcal{D}_{1,1} \cong \mathcal{C}_{2}, \mathcal{D}_{1,1}$ coincides with Kontsevich's eye: its "pupil" $\alpha$ represents the boundary stratum $\mathcal{D}_{2} \times \mathcal{D}_{0,1}$ of codimension 1 , while the point $b$ represents the boundary stratum $\mathcal{C}_{1,0} \times$ $\mathcal{C}_{0,2}^{+} \times \mathcal{D}_{0,1}$ of codimension 2 , graphically

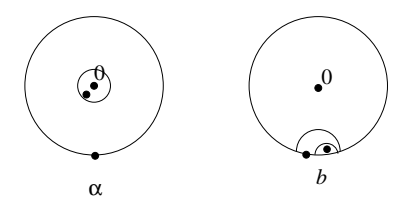

Figure 2. The boundary strata $\alpha$ and $b$ of $\mathcal{D}_{1,1}$.

The subset $\mathcal{Y}_{n, m}^{+}$of $\mathcal{D}_{n, m}^{+}$, for $n \geq 1$, consisting of those configurations, whose projection onto $\mathcal{D}_{1,1}$ belongs to the curve $\ell$, is a smooth submanifold with corners of $\mathcal{D}_{n, m}^{+}$of codimension 1 . Pictorially, a typical configuration in $\mathcal{Y}_{n, 1}$ looks like as follows:

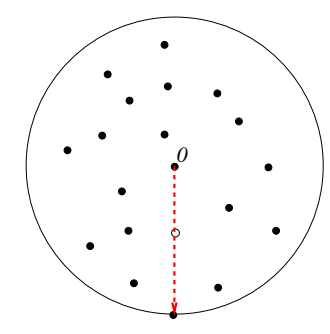

Figure 3. A typical configuration in $\mathcal{Y}_{n, 1}$.

The dashed line represents the curve, along which the first point in $D^{\times}$(labelled by o) moves, going from the origin to the unit circle.

We are interested in the boundary strata of $\mathcal{Y}_{n, 1}$ of codimension 1 , which correspond to the chosen point on the pupil $\alpha$ and to the point $b$ of $\mathcal{D}_{1,1}$, namely

i) configurations in $\mathcal{D}_{n, 1}$, whose projection onto $\mathcal{D}_{1,1}$ is the initial point of the curve $\ell$ (the corresponding strata are denoted collectively by $\left.\mathcal{Y}_{n, 1}^{0}\right)$;

ii) configurations in $\mathcal{D}_{n, 1}$, whose projection onto $\mathcal{D}_{1,1}$ is the final point of the curve $\ell$ (the corresponding strata are denoted collectively by $\left.\mathcal{Y}_{n, 1}^{1}\right)$; 
Let $\gamma$ be a solution of the Maurer-Cartan equation as in (1), $\alpha$ a polyvector field on $V$ of degree -1 and $c=a_{0}$ a Hochschild chain of degree 0 for the algebra $A$.

Proposition 1. For $\gamma, \alpha$ and $c$ as above, the following identities hold true:

$$
\begin{aligned}
& \alpha \cap \mathcal{S}_{\gamma}(c)=\sum_{n \geq 0} \frac{1}{n !} \sum_{\Gamma \in \mathcal{G}_{n+1,1,0}} W_{D, \Gamma}^{0} \mathcal{S}_{\Gamma}(\alpha, \underbrace{\gamma, \ldots, \gamma}_{n}, c), \\
& \mathcal{S}_{\gamma}\left(\mathcal{U}_{\gamma}(\alpha) \cap c\right)=\sum_{n \geq 0} \frac{1}{n !} \sum_{\Gamma \in \mathcal{G}_{n+1,1,0}} W_{D, \Gamma}^{1} S_{\Gamma}(\alpha, \underbrace{\gamma, \ldots, \gamma}_{n}, c),
\end{aligned}
$$

where the weights $W_{D, \Gamma}^{i}, i=0,1$, are defined via

$$
W_{\Gamma}^{i}=\int_{\mathcal{Y}_{n, 1}^{i}} \omega_{D, \Gamma}
$$

Proof. The proof of (11) and (12) relies mainly on the evaluation of the weights $W_{D, \Gamma}^{i}, i=0,1$ : we only give a sketch of such evaluations, referring to [4] for all details.

By construction, all admissible graphs $\Gamma$ appearing in the expressions on the right-hand side of (11) and (12) have all vertices of the first type of valence 2, except the vertex labelled by 1 , which in this case has valence 0 .

First, for any admissible graph $\Gamma$ in $\mathcal{G}_{n+1,1,0}$, the weight $W_{D, \Gamma}^{0}$ vanishes, if 1 has at least one incoming edge: namely, if 1 has one arrow coming from 0 , then the integral vanishes, since the angle form is the derivative of a (locally) constant function. Further, Kontsevich's Vanishing Lemma [7, Lemma 6.4] applies to the remaining cases, whence only the case matters, where 0 and 1 collapse together, and then Lemma 2 does the job. Otherwise, the identity

$$
W_{D, \Gamma}^{0}=W_{D, \Gamma_{0}}
$$

holds true, where $\Gamma_{0}$ is the admissible graph in $\mathcal{G}_{n, 1}$, obtained from $\Gamma$ by collapsing the vertices 0 and 1 . Here is a graphical representation of a general component $Z$ of $\mathcal{Y}_{n+1,1}^{0}$

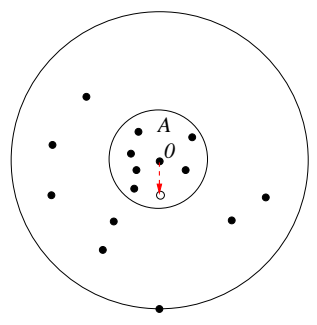

Figure 4. A typical configuration in $\mathcal{Y}_{n, 1}^{0}$.

Second, for an admissible graph $\Gamma$ in $\mathcal{G}_{n+1,1,0}$, the weight $W_{D, \Gamma}^{1}$, restricted to a component $Z$ of $\mathcal{Y}_{n+1,1}^{1}$ of the form

$$
Z \cong \mathcal{C}_{A_{1}, 0} \times \mathcal{C}_{A_{2}, 2}^{+} \times \mathcal{D}_{A_{3}, 1}, \quad 1 \leq\left|A_{1}\right|,
$$

vanishes, unless there are no edges outgoing from $A_{1}$ or from $A_{2}$, in which cases the weight factorizes as

$$
\left.W_{D, \Gamma}^{1}\right|_{Z}=W_{\Gamma_{1}} W_{\Gamma_{2}} W_{D, \Gamma_{3}},
$$

and $\Gamma_{1}$ is the admissible subgraph of $\Gamma$, whose vertices of the first type are labelled by $A_{1}$, $\Gamma_{2}$ is the graph, whose vertices are labelled by $A_{2} \sqcup\{1,2\}$, and obtained by collapsing $\Gamma_{1}$ to the vertex 2 , and $\Gamma_{3}$ is the graph, whose vertices are labelled by $A_{3} \sqcup\{1\}$, obtained by collapsing $\Gamma_{2}$ to the vertex 1 . 
Graphically, a typical configuration in the component $Z$ looks like as

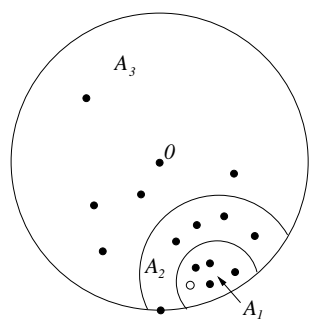

Figure 5. A typical configuration in $\mathcal{Y}_{n, 1}^{1}$.

First of all, if there is an edge e.g. from $A_{1}$ to $A_{2}$, the corresponding contribution vanishes by means of Lemma $2, i$ ); the same argument implies the claim in all other cases. We observe that this also implies that $\Gamma_{i}, i=1,2,3$, is admissible.

Further, the first two factors in the factorization of the weight $W_{D, \Gamma}^{1}$ reduce to usual Kontsevich's weights, again in virtue of Lemma 2,v).

The curve $\ell$ in $\mathcal{D}_{1,1}$ "interpolates" between $\mathcal{Y}_{n+1,1}^{0}$ and $\mathcal{Y}_{n+1,1}^{1}$ : we may evaluate weights of admissible graphs in $\mathcal{G}_{n+1,1,0}$ on the remaining boundary strata of codimension 1 of $\mathcal{Y}_{n+1,1}$, and, by means of Stokes' Theorem, this implies that (11) and (12) coincide at the level of cohomology; for a complete discussion of the corresponding homotopy formula, we refer to [4].

\subsection{The space $\mathcal{D}_{1,2}^{+}$and Hochschild chains of higher degree}

We prove now Theorem 2 in the case, where (3) is applied to Hochschild chains $c$ of higher (negative) degree.

The open unit square in $\mathbb{C}$ can be embedded in the open configuration space $D_{1,2}^{+}$via $(s, t) \mapsto$ $\left[\left(s, 1, e^{2 \pi \mathrm{i} t}\right)\right]$, where the square brackets denote equivalence classes w.r.t. the action of $S^{1}$; we may take a possible closure $\sigma$ of it in the compactification $\mathcal{D}_{1,2}^{+}$as follows:

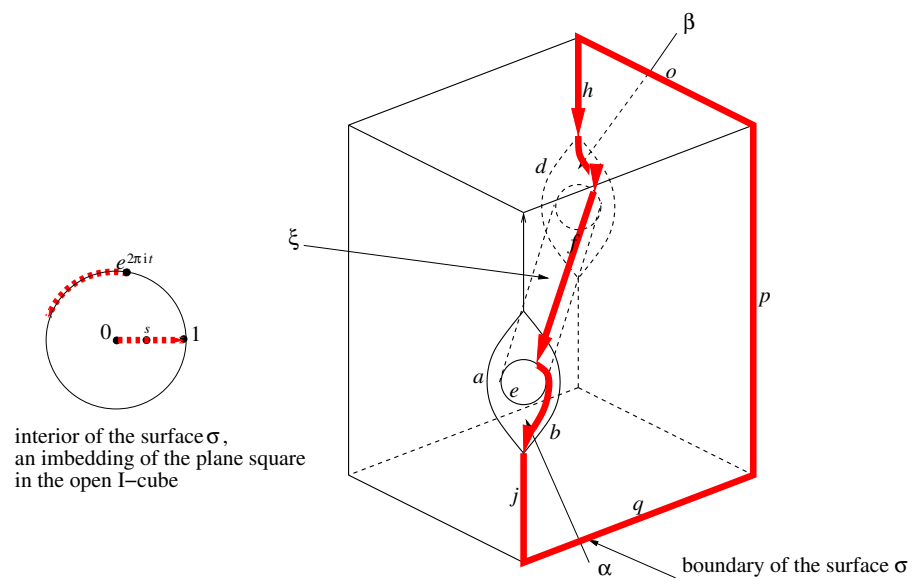

Figure 6. The boundary of $\sigma$ in $\mathcal{D}_{1,2}^{+}$.

Topologically, $\mathcal{D}_{1,2}^{+}$is a "cube with two eyes", or I-cube: we will be mostly interested, in the forthcoming discussion, in the boundary stratum $\xi$ of codimension 1 , which is $\mathcal{D}_{1} \times \mathcal{D}_{0,2}^{+}$, and in the boundary strata $o, q$ of codimension 2 , which are $\mathcal{C}_{1,0} \times \mathcal{C}_{0,2}^{+} \times \mathcal{D}_{0,2}^{+}$and $\mathcal{C}_{1,1} \times \mathcal{C}_{0,2}^{+} \times \mathcal{D}_{0,1}$ respectively; graphically 

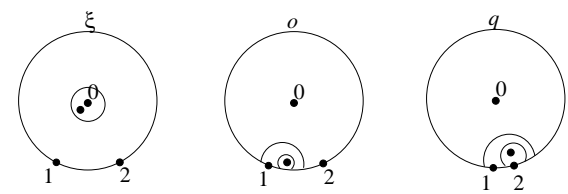

Figure 7. The boundary strata $\xi$, o and $q$ of $\mathcal{D}_{1,2}^{+}$.

We observe that the straight line on the boundary stratum $\xi$ corresponds to the edge $\{s=0\}$, the boundary stratum $o$ corresponds to the edge $\{s=1\}$ and the boundary stratum $q$ corresponds to (a way of imbedding) the point $(1,0)$.

For any two positive integers $(m, n)$, with $n \geq 1$ and $m \geq 2$, the subset $\mathcal{Y}_{n, m}^{+}$of those configurations in $\mathcal{D}_{n, m}^{+}$, whose projection onto $\mathcal{D}_{1,2}^{+}$belongs to $\sigma$, is a smooth, orientable submanifold with corners of $\mathcal{D}_{n, m}^{+}$of codimension 1 . Graphically,

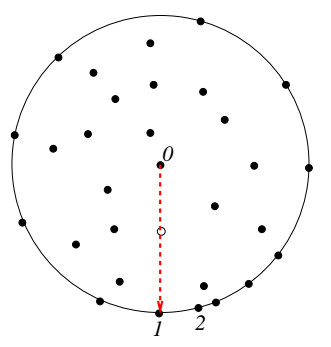

Figure 8. A typical configuration in $\mathcal{Y}_{n, m}^{+}$.

We will need the boundary strata of $\mathcal{Y}_{n, m}^{+}$of codimension 1, corresponding to configurations in $\mathcal{D}_{n, m}^{+}$, whose projection onto the I-cube is in the boundary of $\sigma$ (collectively denoted by $\mathcal{Y}_{n, m}^{+, \partial \sigma}$ ). More precisely, $\mathcal{Y}_{n, m}^{+, \partial \sigma}$ factorizes into eight different types, denoted by $\mathcal{Y}_{n, m}^{+, x}$, and we will consider only $x$ to be the straight line on $\xi$ or $o$ and $q$.

We consider a solution $\gamma$ of the Maurer-Cartan equation as in (1), a polyvector field $\alpha$ on $V$, and a Hochschild chain $c=\left(a_{0}|\cdots| a_{m}\right)$ in $A, m \geq 1$.

Proposition 2. For $\gamma, \alpha$ and $c$ of degree $-m \leq-1$ as above, the following identities hold true:

$$
\alpha \cap \mathcal{S}_{\gamma}(c)=\sum_{n \geq 0} \frac{1}{n !} \sum_{\Gamma \in \mathcal{G}_{n+1, m+1,0}} W_{D, \Gamma}^{\xi} \mathcal{S}_{\Gamma}(\alpha, \underbrace{\gamma, \ldots, \gamma}_{n}, c),
$$

and

$$
\mathcal{S}_{\gamma}\left(\mathcal{U}_{\gamma}(\alpha) \cap c\right)= \begin{cases}\sum_{n \geq 0} \frac{1}{n !} \sum_{\Gamma \in \mathcal{G}_{n+1, m+1,0}} W_{D, \Gamma}^{o} S_{\Gamma}(\alpha, \underbrace{\gamma, \ldots, \gamma}_{n}, c), & |\alpha|=-1, \\ \sum_{n \geq 0} \frac{1}{n !} \sum_{\Gamma \in \mathcal{G}_{n+1, m+1,0}} W_{D, \Gamma}^{q} S_{\Gamma}(\alpha, \underbrace{\gamma, \ldots, \gamma}_{n}, c), & |\alpha| \geq 0,\end{cases}
$$

where the weights $W_{D, \Gamma}^{x}, x=\xi, o, q$, are defined via

$$
W_{D, \Gamma}^{x}=\int_{\mathcal{Y}_{n+1, m}^{+, x}} \omega_{D, \Gamma}
$$

Proof. The proof follows along the same lines of the proof of Proposition 1, with some due changes; once again, we refer to [4] for the complete proofs, while here we make some necessary comments on the degrees, which hold true in this particular situation. 
We observe that, for any admissible graph $\Gamma$ in $\mathcal{G}_{n+1, m, 0}$, the weight $W_{D, \Gamma}^{\xi}$ vanishes, if the vertex 1 has at least one incoming edge, by the very same arguments sketched in the proof of Proposition 1. Otherwise, the identity

$$
W_{D, \Gamma}^{\xi}=W_{D, \Gamma_{0}}
$$

holds true, where $\Gamma_{0}$ in $\mathcal{G}_{n, m, 0}$ is obtained from $\Gamma$ by collapsing the vertices 0 and 1: this generalizes (13) in the proof of Proposition 1, and the proof uses almost the same arguments.

On the other hand, a general component $Z$ of the boundary strata of $\mathcal{Y}_{n, m}^{+, o}$, resp. $\mathcal{Y}_{n, m}^{+, q}$ has the explicit form

$$
\begin{aligned}
& Z \cong \mathcal{C}_{A_{1}, 0}^{+} \times \mathcal{C}_{A_{2}, B_{2}}^{+} \times \mathcal{D}_{A_{3}, B_{3}}^{+}, \quad \text { resp. } \\
& Z \cong \mathcal{C}_{A_{1}, B_{1}}^{+} \times \mathcal{C}_{A_{2}, B_{2}}^{+} \times \mathcal{D}_{A_{3}, B_{3}}^{+},
\end{aligned}
$$

where $A_{i}, i=1,2,3$, are disjoint subsets of $[n]$, with $1 \leq\left|A_{1}\right| \leq n, 0 \leq\left|A_{2}\right| \leq n, 0 \leq\left|A_{3}\right| \leq n$, with $n=\left|A_{1}\right|+\left|A_{2}\right|+\left|A_{3}\right|$, and $B_{i}, i=1,2,3$, are disjoint ordered subsets of $[\mathrm{m}]$ of successive elements, such that $1 \leq\left|B_{1}\right| \leq m, 2 \leq\left|B_{2}\right| \leq m, 1 \leq\left|B_{3}\right| \leq m$, and $m=\left|B_{1}\right|+\left|B_{2}\right|+\left|B_{3}\right|$. Pictorially,
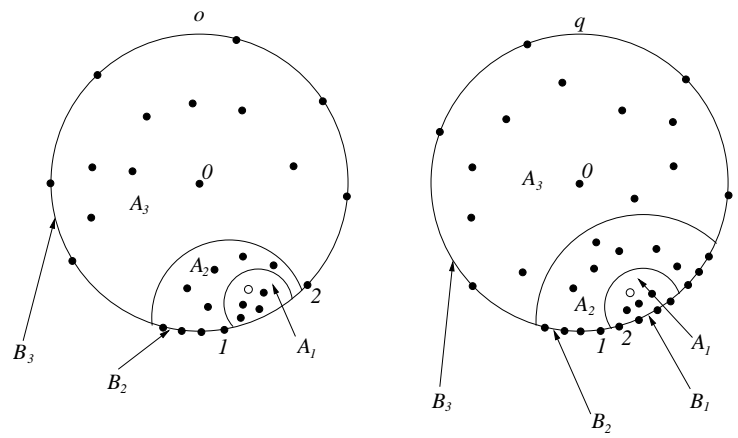

Figure 9. Typical components of $\mathcal{Y}_{n, m}^{+, o}$ and $\mathcal{Y}_{n, m}^{+, q}$.

We consider a component $Z$ of $\mathcal{Y}_{n+1, m}^{o}$ as in (17), resp. of $\mathcal{Y}_{n+1, m}^{q}$ as in (18), and for an admissible graph $\Gamma$ as before, we denote by $\Gamma_{1}$ the admissible subgraph of $\Gamma$, whose vertices are labelled by $A_{1} \sqcup B_{1}$, by $\Gamma_{2}$ the graph, whose vertices are labelled by $A_{2} \sqcup B_{2}$, and obtained by collapsing $\Gamma_{1}$ to a single vertex, and by $\Gamma_{3}$ the graph, whose vertices are labelled by $A_{3} \sqcup B_{3}$, obtained by collapsing $\Gamma_{2}$ to a single vertex.

The weight $W_{D, \Gamma}^{o}$, restricted to a component $Z$ of $\mathcal{Y}_{n+1, m}^{+, o}$ as above vanishes, unless there are no edges going from $A_{1}$ to $A_{2}$ or $A_{3}$, or from $A_{2}$ to $A_{3},\left|B_{2}\right|=2$, and $\alpha$ has degree -1 . In fact, the weight $W_{D, \Gamma}^{o}$ factorizes as

$$
\left.W_{D, \Gamma}^{o}\right|_{Z}=W_{\Gamma_{1}} W_{\Gamma_{2}} W_{D, \Gamma_{3}},
$$

with $B_{1}=\varnothing$. In particular, the third weight on the right-hand side is non-trivial only if $2\left|A_{1}\right|-2$ equals the degree of the integrand, which, by the above reasonings, is precisely $2\left(\left|A_{1}\right|-1\right)+|\alpha|+1$ (since all vertices of the first type in $A_{1}$ are 2-valent except the first vertex): therefore, the integral is non-trivial only if $|\alpha|=-1$.

The weight $W_{D, \Gamma}^{q}$, restricted to a component $Z$ of $\mathcal{Y}_{n+1, m}^{+, q}$ as above vanishes, unless there are no edges going from $A_{1}$ to $A_{2}$ or $A_{3}$, or from $A_{2}$ to $A_{3},\left|B_{2}\right|=2$, and $\left|B_{1}\right|=|\alpha|+1$ (which implies that $\alpha$ has degree bigger or equal than 0$)$. In such cases, the weight $W_{D, \Gamma}^{o}$ factorizes as

$$
W_{D, \Gamma}^{o} \mid Z=W_{\Gamma_{1}} W_{\Gamma_{2}} W_{D, \Gamma_{3}},
$$


with $1 \leq\left|B_{1}\right|$. Dimensional reasons as in the case of a component $Z$ of $\mathcal{Y}_{n+1, m}^{+, q}$ force the degree of $\alpha$ to be bigger or equal than 0: namely, the degree of the third integrand is $2\left(\left|A_{1}\right|-1\right)+|\alpha|+1$, and the integral is non-trivial only if it equals $2\left|A_{1}\right|+\left|B_{1}\right|-2$. Since $1 \leq\left|B_{1}\right|$, the non-triviality condition forces $|\alpha|=\left|B_{1}\right|-1$, whence the claim.

This result obviously generalizes (14) in the proof of Proposition 1, and its proof is the same as the proof of (14).

The surface $\sigma$ "interpolates" between the boundary strata $\mathcal{Y}_{n+1, m}^{+, \xi}, \mathcal{Y}_{n+1, m}^{+, o}$ and $\mathcal{Y}_{n+1, m}^{+, q}$ : the "interpolation" in this situation is of course more complicated than the one in Subsection 5.1, since we have to keep track of the boundary of $\sigma$. In fact, the weighted sums as in (15) and (16), where we integrate over boundary strata of $\mathcal{Y}_{n+1, m}^{+, x}, x=h, j, p$, vanish; the weighted sums over the remaining boundary strata of $\mathcal{Y}_{n+1, m}^{+}$can be also explicitly evaluated, and, by means of Stokes' Theorem, they produce a homotopy formula, proving that the left-hand sides of (15) and (16) coincide at the level of cohomology.

\section{Duflo isomorphism on (co)invariants revisited}

Let $\mathfrak{g}$ be a finite dimensional Lie algebra over $\mathbb{C}$.

First of all, the (modified) Duflo element [6] is defined via

$$
J:=\operatorname{det}\left(\frac{e^{\mathrm{ad} / 2}-e^{-\mathrm{ad} / 2}}{\mathrm{ad}}\right) \in \widehat{\mathrm{S}}\left(\mathfrak{g}^{*}\right)^{\mathfrak{g}} .
$$

We have a morphism of $\mathfrak{g}$-modules

$$
\mathcal{D}:=\operatorname{sym} \circ\left(J^{1 / 2} \cdot\right): \mathrm{S}(\mathfrak{g}) \longrightarrow \mathrm{U}(\mathfrak{g}),
$$

where $U(\mathfrak{g})$ is the Universal Enveloping Algebra of $\mathfrak{g}$, and sym denotes the usual symmetrization map from the symmetric algebra $S(\mathfrak{g})$ to $U(\mathfrak{g})$.

The following result generalizes to coinvariants the well-known Duflo isomorphism [6].

Theorem 5. The map $\mathcal{D}$ restricts to an isomorphism of algebras $\mathrm{S}(\mathfrak{g})^{\mathfrak{g}} \stackrel{\sim}{\longrightarrow} \mathrm{U}(\mathfrak{g})^{\mathfrak{g}}=\mathcal{Z}(\mathrm{U}(\mathfrak{g}))$ on invariants, and induces an isomorphism of $\mathrm{S}(\mathfrak{g})^{\mathfrak{g}}$-modules $\mathrm{S}(\mathfrak{g})_{\mathfrak{g}} \stackrel{\sim}{\longrightarrow} \mathrm{U}(\mathfrak{g})_{\mathfrak{g}}=\mathcal{A}(\mathrm{U}(\mathfrak{g}))$ on coinvariants.

Here $\mathcal{Z}(B)$ denotes the center of an algebra $B$, and $\mathcal{A}(B)=B /[B, B]$ its abelianization.

We sketch here a proof of Theorem 5 in the spirit of the approach of $[7,9]$ to the original Duflo isomorphism.

We consider the Kirillov-Kostant-Souriau Poisson bivector $\pi$ on $\mathfrak{g}^{*}$ and associated product $\star$. Since the product $\star$ obeys $x \star y-y \star x=[x, y]_{\mathfrak{g}}{ }^{2}$, viewing $x, y \in \mathfrak{g}$ as linear functions on $\mathfrak{g}^{*}$, there is an algebra morphism

$$
\mathcal{I}: \mathrm{U}(\mathfrak{g}) \longrightarrow(\mathrm{S}(\mathfrak{g}), \star) ; x \longmapsto x .
$$

The map $\mathcal{I}^{-1} \circ \mathcal{U}_{\gamma}$ induces an algebra isomorphism by means of Theorem 1, Section 2,

$$
\mathrm{S}(\mathfrak{g})^{\mathfrak{g}}=\mathcal{Z}(\mathrm{S}(\mathfrak{g}),\{,\}) \longrightarrow \mathcal{Z}(\mathrm{U}(\mathfrak{g}))=\mathrm{U}(\mathfrak{g})^{\mathfrak{g}}
$$

while, dually, $\mathcal{S}_{\gamma} \circ \mathcal{I}$ induces an isomorphism of $\mathrm{S}(\mathfrak{g})^{\mathfrak{g}}$-modules by means of Theorem 2, Section 2,

$$
\mathrm{U}(\mathfrak{g})_{\mathfrak{g}}=\mathcal{A}(\mathrm{U}(\mathfrak{g})) \longrightarrow \mathcal{A}(\mathrm{S}(\mathfrak{g}),\{,\})=\mathrm{S}(\mathfrak{g})_{\mathfrak{g}}
$$

\footnotetext{
${ }^{2}$ We may set $\hbar=1$, as the Poisson structure is linear.
} 
In [11], the restriction of $\mathcal{U}_{\gamma}$ to $\mathrm{S}(\mathfrak{g})$ has been shown to be the identity, which, coupled with Kontsevich's discussion [7, Section 8], implies that $\mathcal{I}^{-1} \circ \mathcal{U}_{\gamma}=\mathcal{I}^{-1}=\mathcal{D}: \mathrm{S}(\mathfrak{g}) \rightarrow \mathrm{U}(\mathfrak{g})$.

Shoikhet's proof of the fact that the restriction of $\mathcal{U}_{\gamma}$ to functions is the identity can be summarized as follows: for any function $f \in \mathrm{S}(\mathfrak{g}), \mathcal{U}_{\gamma}(f)$ is expressed only via so-called inner wheels, whose weights vanish by the main result of [11]. Pictorially, an inner wheel looks like as follows:

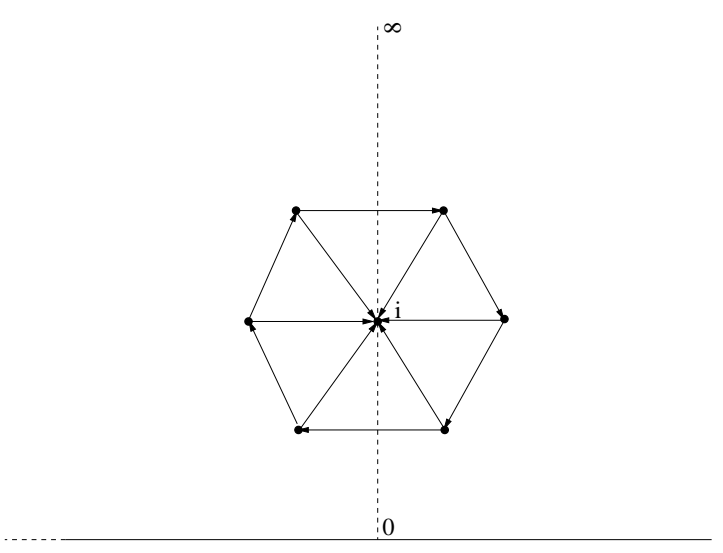

Figure 10. An inner wheel.

Theorem 5 follows then from the following

Proposition 3. The restriction of $\mathcal{S}_{\gamma}$ on $\mathrm{S}(\mathfrak{g})$ coincides with the identity map.

Proof. By the arguments of [10, Paragraph 3.6.1], the only admissible graphs contributing non-trivially to $\mathcal{S}_{\gamma}$ are of the form
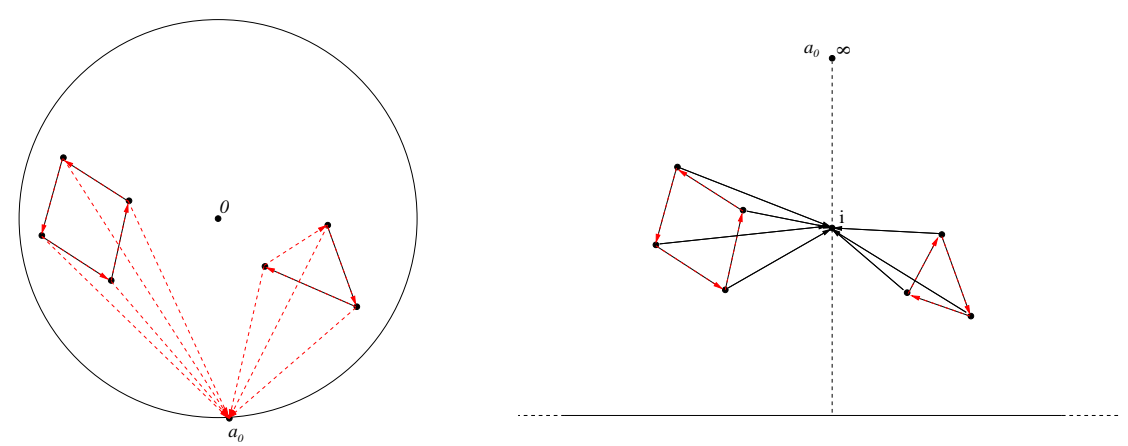

Figure 11. A typical admissible graph in $\mathcal{S}_{\gamma}$ on functions and its counterpart in the upper half-plane.

Further, we use a correspondence between weights of admissible graphs in $\mathcal{G}_{n, 1,0}$ and admissible graphs in $\mathcal{G}_{n+1,0}$, by means of the Möbius transformation

$$
\psi: \mathcal{H} \sqcup \mathbb{R} \longrightarrow D \sqcup S^{1} \backslash\{1\} ; \quad z \longmapsto \frac{z-i}{z+i},
$$

which induces (see Subsection 3.3) isomorphisms $\mathcal{C}_{n+1,0} \stackrel{\sim}{\longrightarrow} \mathcal{D}_{n, 1}$, to prove that $\mathcal{S}_{\gamma}=$ id.

First of all, given on $\mathcal{D}_{1,1}$ the 1-form $\omega_{D}$ as in Lemma 2, then the 1-form $\omega:=\psi^{*} \omega_{D}$ on $\mathcal{C}_{2,0}$ is a difference of usual Kontsevich's angle forms as in Lemma 1 . Then the weight $W_{D, \Gamma}$ of an admissible graph $\Gamma$ in $\mathcal{G}_{n, 1,0}$ is pulled-back to a weight $W_{\Gamma^{\prime}}, \Gamma^{\prime}$ being admissible in $\mathcal{G}_{n+1,0}$ : the vertex 0 is mapped to a vertex of the first type, while the only vertex of the second type is mapped to $\infty$ in the complex upper half-plane. The factors of $\omega_{D, \Gamma}$ are pulled-back to $i$ ) usual 
forms $\omega_{e}$, whenever $e$ is an edge from some vertex (of the first and of the second type) to 1, and the "new" $e$ is now an edge from the (inverse image of the) starting point to $\mathrm{i}$, and $i i$ ) differences between $\omega_{e}$ and $\omega_{e(\mathrm{i})}$, if $e$ is an edge between two vertices (of the first and second type, neither of which is 0 ) and the new edge $e$ connects the (inverse images of the) endpoints, while $e(\mathrm{i})$ is an edge, whose starting point is the starting point of $e$ and whose endpoint is i. We have used here the arguments exposed in Appendix 1 of [4], to which we refer for a more detailed discussion, as well as for the properties of $\omega_{D}$. We also used the fact that the form $\omega$ vanishes when its final point goes to infinity, which finally implies the above correspondence.

We observe that dashed arrows denote forms $\omega_{D, e}$ on $\mathcal{D}_{n, 1}$ in the left-most graph of Fig. 11, while we have used plain, resp. dashed, arrows to denote forms $\omega_{e}$ on $\mathcal{C}_{n+1,0}$, resp. differences of such forms, in the right-most graph.

Finally, we use the following graphical calculus for replacing dashed edges by plain ones:

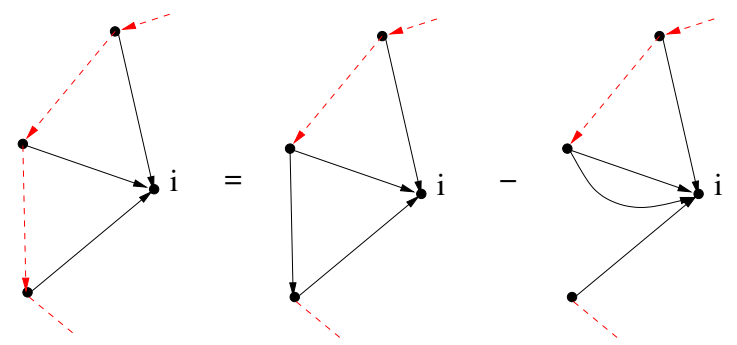

Figure 12. Replacing dashed edges by plain ones in an inner wheel.

The second graph on the right-hand side has a double edge, whence its weight vanishes. Thus, the Shoikhet weight of the left-most graph in Fig. 11 equals the usual Kontsevich weight of the right-most one (i.e. with all edges turned into black ones), which is zero by [11].

We finally observe that Proposition 3 has been proved in Subsubsection 3.6.2 of [10] under the assumption of the validity of Conjecture 3.5.3.1, which is implied by Theorem 2: our proof, on the contrary, is purely based on the main result of [11] and on the properties of the forms $\omega_{D}$.

\section{Conclusion}

In this paper, we have proved Shoikhet's conjecture using configuration space integrals: it is worthwhile noticing that we did not exploit all boundary strata of the I-cube, which replaces in our homotopy argument Kontsevich's eye (namely, we made use only of the boundary stratum $\xi$ of codimension 1 and of the boundary strata $o$ and $q$ of codimension 2). In fact, Shoikhet's conjecture can be viewed as a special case of a more general result, which involves a MaurerCartan element of a more general shape, i.e. a sum of polyvector fields of different degrees, to which, via Kontsevich's formality, corresponds also a sum of polydifferential operators, also of different degrees: accordingly, all boundary strata of the surface $\sigma$ in the I-cube contribute to the proof of this more general result [4].

\section{Acknowledgement}

We thank Giovanni Felder for useful discussions and comments. The research of D.C. (on leave of absence from Université Lyon 1) is fully supported by the European Union thanks to a Marie Curie Intra-European Fellowship (contract number MEIF-CT-2007-042212). 


\section{References}

[1] Arnal D., Manchon D., Masmoudi M., Choix des signes pour la formalité de M. Kontsevich, Pacific J. Math. 203, (2002), 23-66, math.QA/0003003.

[2] Bayen F., Flato M., Fronsdal C., Lichnerowicz A., Sternheimer D., Deformation theory and quantization. I. Deformations of symplectic structures, Ann. Physics 111 (1978), 61-110.

Bayen F., Flato M., Fronsdal C., Lichnerowicz A., Sternheimer D., Deformation theory and quantization. II. Physical applications, Ann. Physics 111 (1978), 111-151.

[3] Calaque D., Rossi C.A., Lectures on Duflo isomorphisms in Lie algebras and complex geometry, http://math.univ-lyon1.fr/ calaque/LectureNotes/LectETH.pdf.

[4] Calaque D., Rossi C.A., Compatibility with cap-products in Tsygan's formality and homological Duflo isomorphism, arXiv:0805.3444.

[5] Cattaneo A.S., Keller B., Torossian Ch., Bruguières A., Déformation, quantification, théorie de Lie, Panoramas et Synthèses, Vol. 20, SMF, Paris, 2005.

[6] Duflo M., Opérateurs différentiels bi-invariants sur un groupe de Lie, Ann. Sci. École Norm. Sup. (4) 10 (1977), 265-288.

[7] Kontsevich M., Deformation quantization of Poisson manifolds, Lett. Math. Phys. 66 (2003), 157-216, q-alg/9709040.

[8] Manchon D., Torossian Ch., Cohomologie tangente et cup-produit pour la quantification de Kontsevich, Ann. Math. Blaise Pascal 10 (2003), 75-106, math.QA/0106205.

[9] Pevzner M., Torossian Ch., Isomorphisme de Duflo et la cohomologie tangentielle, J. Geom. Phys. 51 (2004), 487-506, math.QA/0310128.

[10] Shoikhet B., A proof of the Tsygan formality conjecture for chains, Adv. Math. 179 (2003), 7-37, math.QA/0010321.

[11] Shoikhet B., Vanishing of the Kontsevich integrals of the wheels, in EuroConférence Moshé Flato 2000, Part II (Dijon), Lett. Math. Phys. 56, (2001), 141-149, math.QA/0007080.

[12] Tamarkin D., Tsygan B., Cyclic formality and index theorems, EuroConférence Moshé Flato 2000, Part II (Dijon), Lett. Math. Phys. 56 (2001), 85-97.

[13] Tsygan B., Formality conjectures for chains, in Differential Topology, Infinite-Dimensional Lie Algebras, and Applications, Amer. Math. Soc. Transl. Ser. 2, Vol. 194, Amer. Math. Soc., Providence, RI, 1999, 261-274, math.QA/9904132. 